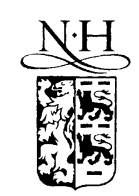

ELSEVIER
Computer methods in applied mechanics and engineering

www.elsevier.com/locate/cma

\title{
Finite element analysis of particle motion in steady inspiratory airflow
}

\author{
T.W.H. Sheu* ${ }^{*}$ S.K. Wang, S.F. Tsai \\ Department of Engineering Science and Ocean Engineering, National Taiwan University, 73 Chou-Shan Road, Taipei, Taiwan, ROC
}

Received 10 May 2000; received in revised form 14 January 2001

\begin{abstract}
To accurately model the inhaled particle motion, equations governing particle trajectories in carrier flow are solved together with the Navier-Stokes equations. Under the relatively dilute particle condition in the mixture, equations for two phases are coupled through the interface drag shown in the solid-phase momentum equations. The present study investigates bifurcation flow in the human central airway using the finite element method. In the gas phase, we employ the biquadratic streamline upwind Petrov-Galerkin finite element model to simulate the incompressible air flow. To solve the equations of motion for the inhaled particles, we apply another biquadratic streamline upwind finite element model. A feature common to two models applied to each phase of equations is that both of them provide nodally exact solutions to the convection-diffusion and the convection-reaction equations, which are prototype equations for the gasphase and the solid-phase equations, respectively. In two dimensions, both models have ability to introduce physically meaningful artificial damping terms solely in the streamline direction. With these terms added to the formulation, the discrete system is enhanced without compromising the numerical diffusion error. Tests on inspiratory problem were conducted, and the results are presented, with an emphasis on the discussion of particle motion. () 2002 Elsevier Science B.V. All rights reserved.
\end{abstract}

Keywords: Dilute particle condition; Two phases; Interface drag; Convection-diffusion; Convection-reaction

\section{Introduction}

The human lung respiratory system is a large-scale mechanical system having 23 branching airways. These airways have different diameters, ranging from $18 \mathrm{~mm}$ for the trachea to $0.4 \mathrm{~mm}$ for other posterior branchea [1]. Besides difficulties with geometrical complexities and equation nonlinearities in the numerical simulation, this system is physiologically very complex. Breathed air ventilates towards the alveolar and then diffuses into capillary blood. The resulting arterialized blood is carried by the pulmonary circulation. A complete understanding of the above lung respiration system through numerical investigation involves simulating air and blood flows in vessels, subject to tissue deformation. To make the analysis simple, we

\footnotetext{
${ }^{*}$ Corresponding author. Tel.: +886-2-2362-5470x246; fax: +886-2-2392-9885.

E-mail address: sheu@sccs.na.ntu.edu.tw (T.W.H. Sheu).
} 
concentrate in this paper on the gas flow in the airway without considering the effect of vessel elasticity on the flow motion. A report on our investigation of blood flow in pulmonary circulation has been published elsewhere $[2,3]$.

The respiratory system is characterized by a network of dichotomous bronchia. For this reason, the study of airflow in bifurcation airways was considered to be a first step towards gaining a better understanding of the human inspiratory/expiratory system. Investigating inspiratory and expiratory airway flows, without doubt, can be accomplished using scaled-up laboratory models. Such experiments are, however, expensive and time consuming. Owing to rapid advances in computer technology and ever-improving numerical techniques, we were motivated to exploit the computational fluid dynamics technique as a tool for gaining a better understanding of lung respiration. An additional advantage gained is that numerical simulation allows investigation of various modelling conditions, which may not be possible in a laboratory setting, at reduced expenditures in time and in cost.

Study of flows in a branching airway has long been the subject of interest for many reasons. The main reason lies in the tendency for circulation regions to form in the airway. These recirculating eddies can, in turn, entrain inhaled particles. Therefore, developing an analysis code capable of accurately predicting flow reversal and deposition sites of inhaled particles in the respiratory airway is important if we are to evaluate the convective transport of particles in the human lung. Several models of deposition have been proposed in the literature. Interested readers are referred to a recent review paper for additional details about the respiratory tract deposition models [4]. Accurate prediction of particle trajectories in the model configurations was another focus of the present study.

The rest of this paper is organized as follows: In Section 2, we present Navier-Stokes equations for the airflow. These equations of motion, subject to divergence-free velocity constraint condition, are solved together with the solid-phase equations of motion, namely, the particle trajectory equations. For the elliptic equations to be well-posed, boundary conditions should be prescribed. Section 3 presents the weighted residuals statement for the gas-phase equations. It is shown that the case with pressure prescribed a priori at the vessel exit can be theoretically modelled. This is followed by an introduction to the finite elements used and application of the upwind model to resolve pressure and velocity oscillations, respectively. In Section 4, we describe the test problem in greater detail. The results obtained from studies of a bifurcation configuration are presented in Section 5. Finally, we draw conclusions in Section 6.

\section{Governing equations}

In the presence of inhaled particles, the biomechanical study of lung respiration, in essence, involves investigating gas-solid two-phase equations. Like many engineering analyses, assumptions and simplifications are made in order to formulate a computationally tractable problem. In what follows, the gas-solid mixture is assumed to have a constant solid-phase density $\rho_{\mathrm{p}}$. As for the inhaled air, we consider that the air speed is not so high as to permit avoiding the need to choose an appropriate, if any, turbulence model for the airflow. Like many previous investigations, such as some of them in [1-7], the flow is investigated in a domain of two dimensions to facilitate the analysis. As with the conventional gas-solid two-phase flow analyses, we assume that the gas and solid phases have complementary regions. In each region, the material may be treated as a homogeneous continuum. The typical quantity used to describe such a mixture is known as the porosity $\alpha$, defined as the ratio of the volume occupied by the gas phase to the total volume. For the present study, the inhaled fine particles are so diluted that their volumes are considered to be negligibly small. As a result, it is rational to assume that $\alpha=1$ in the present analysis.

Under the assumed condition for the porosity in the gas-solid mixture, we can use the Favre-averaging (or mass-weighted averaging) approach [8], which has been successfully applied in our previous studies of gas-solid two-phase flow in gun ballistics [9-11]. The assumption of flow incompressibility is also made 
since the Mach number in the airway is much less than 0.2. With these assumptions and approximations in mind, the solid-phase continuity equation is automatically satisfied and is, thus, not needed. Under the laminar and incompressible conditions, the Favre-averaging governing equations for a two-phase inspiratory/respiratory flow problem are summarized separately below:

Gas-phase continuity

$$
\nabla \cdot \underline{u}_{\mathrm{a}}=0 .
$$

Gas-phase momentum

$$
\frac{\partial \underline{u}_{\mathrm{a}}}{\partial t}+\underline{u}_{\mathrm{a}} \cdot \nabla \underline{u}_{\mathrm{a}}=-\frac{1}{\rho_{\mathrm{a}}} \nabla p+\frac{1}{\rho_{\mathrm{a}}} \nabla \cdot \underline{\pi}+\underline{u}_{\mathrm{p}} \Gamma
$$

where $\Gamma$ is the interfacial mass transfer. Since chemical reaction is not invoked in between inhaled air and particles, there exists no regression rate of the solid phase. Thus, the value of $\Gamma$ is rationally assigned to be zero. As for $\underline{\underline{\pi}}$, it represents the stress tensor in the gas phase

$$
\underline{\underline{\pi}}=2 \mu_{\mathrm{a}} \underline{\underline{D}} \text {. }
$$

In the above, $\underline{\underline{D}}$ represents the deformation tensor (or rate of strain tensor). The subscripts "a" and "p" in Eqs. (1) and (2) denote the air and particle, respectively.

Solid-phase momentum

$$
\frac{\partial \underline{u}_{\mathrm{p}}}{\partial t}+\underline{u}_{\mathrm{p}} \cdot \nabla \underline{u}_{\mathrm{p}}=-\frac{1}{\rho_{\mathrm{p}}} \nabla p+\frac{1}{\rho_{\mathrm{p}}} \nabla \cdot \underline{\underline{R}}+\frac{S_{\mathrm{p}}}{\rho_{\mathrm{p}} V_{\mathrm{p}}}\langle\vec{F}\rangle-\frac{\underline{u}_{\mathrm{p}} \Gamma}{\rho_{\mathrm{p}}} .
$$

In Eq. (4), $S_{\mathrm{p}}\left(\equiv 4 \pi r_{\mathrm{p}}^{2}\right)$ and $V_{\mathrm{p}}\left(\equiv \frac{4}{3} \pi r_{\mathrm{p}}^{3}\right)$ are the surface area and volume of an inhaled particle with a mean particle radius of $r_{\mathrm{p}}$. Another important source term for determining the particle velocity $\underline{u}_{\mathrm{p}}$ is the interphase drag $\langle\vec{F}\rangle^{i}$. This drag, appearing only in multiple phases, is produced owing to the ineligible relative velocity, $\underline{u}_{\mathrm{R}}\left(\underline{\underline{u}}_{\mathrm{a}}-\underline{u}_{\mathrm{p}}\right)$, between two phases. As a result, it is conventional to represent $\langle\vec{F}\rangle$ by

$$
\langle F\rangle=\frac{\rho_{\mathrm{a}}\left|\underline{u}_{\mathrm{R}}\right| \underline{u}_{\mathrm{R}}}{6} \hat{f}
$$

The constitutive equation for the drag coefficient $\hat{f}$ follows the one given by Wallis [12]. Another source term $\underline{R}$ in Eq. (4) represents the granular stress tensor. This tensor is classified as a diagonal tensor, i.e., $\underline{R}=\underline{R_{\mathrm{p}}} \underline{\underline{I}}$, where $\underline{\underline{I}}$ denotes the identity tensor. Usually, the intergranular stress magnitude $R_{\mathrm{p}}$ depends on the critical porosity [13]. Since porosity for the present mixture is by all means larger than the chosen critical value, there is no direct contact between particles. This implies that $R_{\mathrm{p}}=0$.

Taking all the above considerations into account, the working equation for modelling the particle trajectory is as follows:

$$
\underline{u}_{\mathrm{p}} \cdot \nabla \underline{u}_{\mathrm{p}}=-\frac{1}{\rho_{\mathrm{p}}} \nabla p+C_{p i}\left(\underline{u}_{\mathrm{a}}-\underline{u}_{\mathrm{p}}\right),
$$

where $C_{p i}$ has a close relevance to the Stokes number defined in [14]

$$
C_{p i}=\frac{3 \rho_{\mathrm{a}}}{8 \rho_{\mathrm{p}} r_{\mathrm{p}}}\left|\underline{u}_{\mathrm{a}}-\underline{u}_{\mathrm{p}}\right| C_{\mathrm{D}}
$$

The Reynolds number has a considerable effect on the drag coefficient $C_{\mathrm{D}}$, as suggested by Cliff and Gauvin [15]. Thus, we are led to employ the following constitutive equation for $C_{\mathrm{D}}$ :

$$
C_{\mathrm{D}}=\frac{24}{R e_{\mathrm{p}}}\left(1+0.15 R e_{\mathrm{p}}^{0.687}\right),
$$

where the particle Reynolds number $R e_{\mathrm{p}}$ is defined by 


$$
R e_{\mathrm{p}}=\frac{2 \rho_{\mathrm{a}}\left|\underline{u}_{\mathrm{a}}-\underline{u}_{\mathrm{p}}\right| r_{\mathrm{p}}}{\mu_{\mathrm{a}}}
$$

Eq. (8) is suggested for use when $R e_{\mathrm{p}}<10^{3}$. Given the above particle Reynolds number, the source term $C_{p i}$ can be rewritten as

$$
C_{p i}=\frac{3 \mu_{\mathrm{a}} R e_{\mathrm{p}} C_{\mathrm{D}}}{16 \rho_{\mathrm{p}} r_{\mathrm{p}}^{2}}
$$

When formulating Eq. (6) for particle motion in carrier gas flow, we neglect the Saffman lift force [16] and the Brownian force [17]. For particles that are not too small and for flows with high shear rates, the Saffman lift force can be considered to be prevailing in carrier flow. As for the Brownian force, it is important only for submicron particles. Gravitational force is not considered in cases of negligibly small free convection. The so-called Stokes-Cunningham slip correction factor $[14,16]$ is not modelled here since the molecular mean free-path in carrier gas flow is fairly small under the investigated conditions.

Before presenting the numerical method, we summarize first the following equations which form the basis for solving the field variables $\underline{u}_{\mathrm{a}}, \underline{u}_{\mathrm{p}}$ and $p$ :

$$
\begin{aligned}
& \nabla \cdot \underline{u}_{\mathrm{a}}=0, \\
& \underline{u}_{\mathrm{a}} \cdot \nabla \underline{u}_{\mathrm{a}}=-\frac{1}{\rho_{\mathrm{a}}} \nabla p+\frac{\mu_{\mathrm{a}}}{\rho_{\mathrm{a}}} \nabla^{2} \underline{u}_{\mathrm{a}}, \\
& \underline{u}_{\mathrm{p}} \cdot \nabla \underline{u}_{\mathrm{p}}=-\frac{1}{\rho_{\mathrm{p}}} \nabla p+\frac{3 \rho_{\mathrm{a}}}{8 \rho_{\mathrm{p}} r_{\mathrm{p}}}\left|\underline{u}_{\mathrm{a}}-\underline{u}_{\mathrm{p}}\right|\left(\underline{u}_{\mathrm{a}}-\underline{u}_{\mathrm{p}}\right) C_{\mathrm{D}} .
\end{aligned}
$$

Provided that $\underline{u}_{\mathrm{a}}$ is computed from the gas-phase equations, Eq. (13) is well posed. As a result, the above formulation has a benefit in the sense that two fields of equations can be solved separately and, thus, considerably simplifying the analysis. We can refer to Eq. (13) as the particle trajectory equation for determining the deposition sites of particles inhaled in the airway. This equation needs to be accurately solved since deposition sites have been known for years to be essential for determining the efficiency of aerosolized medicines.

\section{Finite element model for gas flow equations}

For wider future application, equations for modelling airflow are made dimensionless through the user's chosen reference length, $L$, and the characteristic velocity, $U$. The resulting dimensionless equations are expressed as follows:

$$
\begin{aligned}
& \underline{u}_{\mathrm{a}} \cdot \nabla \underline{u}_{\mathrm{a}}=-\nabla p+\frac{1}{\operatorname{Re}} \nabla^{2} \underline{u}_{\mathrm{a}}, \\
& \nabla \cdot \underline{u}_{\mathrm{a}}=0 .
\end{aligned}
$$

The Reynolds number shown above is defined as $R e=U L / v$, where $v$ is the kinematic viscosity of the airflow.

In this study, we seek to obtain the steady-state solutions of the Navier-Stokes and continuity equations using a method suited to solving complex geometry problems. Calculations exhibiting the divergence-free property on the velocity field are desired. For this reason, we adopt the coupled solution algorithm. In the context of the weighted residuals method, we denote by $L^{2}(\Omega)$ the functional space that is square integrable 
over $\Omega$. We also define its constrained space $L^{2}(\Omega)\left(\equiv\left\{q \in L^{2}(\Omega)=\int_{\Omega} q \mathrm{~d} \Omega=0\right\}\right)$, which consists of square integrable functions having zero mean over $\Omega$, and the Sobolev space $H^{1}(\Omega)\left(\equiv\left\{q \in L^{2}(\Omega): D q \in L^{2}(\Omega)\right\}\right)$. Here, $D$ denotes the derivative of order 1 . In addition, the subspace of $H^{1}(\Omega)$, namely $H_{0}^{1}(\Omega)$, is needed for constructing a weighted residuals statement. By definition, all the elements in $H_{0}^{1}(\Omega)\left(\equiv\left\{q \in H^{1}(\Omega)\right.\right.$ : $q=0$ on $\Gamma\}$ ) have one square integrable derivative over $\Omega$, and their values vanish on the boundary $\Gamma$.

Given the admissive function $\underline{w} \in H_{0}^{1}(\Omega) \times H_{0}^{1}(\Omega)\left(\equiv \mathbf{H}_{0}^{1}\right)$ and the pressure mode $q \in L_{0}^{2}(\Omega)(\equiv \mathbf{P})$, we can find solutions of $(\underline{u}, p) \in V\left(\equiv \mathbf{H}_{0}^{1} \times \mathbf{P}\right)$ using the following equations:

$$
\begin{aligned}
& \int_{\Omega}\left(\underline{u}_{\mathrm{a}} \cdot \nabla\right) \underline{u}_{\mathrm{a}} \cdot \underline{w} \mathrm{~d} \Omega+\frac{1}{R e} \int_{\Omega} \nabla \underline{u}_{\mathrm{a}}: \nabla \underline{w} \mathrm{~d} \Omega-\int_{\Omega} p \nabla \cdot \underline{w} \mathrm{~d} \Omega=\int_{\Gamma / \Gamma_{n}} r \underline{w} \cdot \underline{n} \mathrm{~d} \Gamma+\int_{\Gamma / \Gamma_{r}} \underline{s} \cdot \underline{w} \times \underline{n} \mathrm{~d} \Gamma, \\
& \int_{\Omega}\left(\nabla \cdot \underline{u}_{\mathrm{a}}\right) q \mathrm{~d} \Omega=0,
\end{aligned}
$$

where $\underline{n}$ denotes the unit normal vector to $\Gamma$ and

$$
\begin{aligned}
& -p+\frac{1}{R e} \underline{n} \cdot \nabla \underline{u}_{\mathrm{a}} \cdot \underline{n}=r \quad \text { on } \Gamma / \Gamma_{n},
\end{aligned}
$$

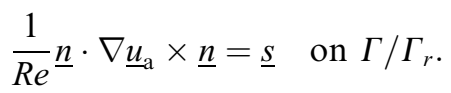

To specify boundary conditions, we denote here two boundary segments of $\Gamma$ as $\Gamma_{n}$ and $\Gamma_{r}$. Note that $\Gamma / \Gamma_{i}(i=n, r)$, shown in Eqs. (18) and (19), are defined as complements of $\Gamma_{i}$ in $\Gamma$. This implies that if $\phi \in \Gamma / \Gamma_{i}$, then $\phi \in \Gamma$ but $\phi \notin \Gamma_{i}$. In the above, $\underline{w}\left(\in H_{0}^{1}(\Omega) \times H_{0}^{1}(\Omega)\right)$ and $q\left(\in L_{0}^{2}(\Omega)\right)$ two test functions for

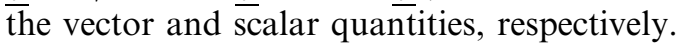

In the finite element computation of incompressible flows there exist two sources of potential numerical instabilities. One is due to an inappropriate combination of interpolation functions used for the velocity and pressure: $\underline{u}^{h}=\sum u_{i}^{h} N_{i}^{h}$ and $p^{h}=\sum p_{i}^{h} M_{i}^{h}$, where $\left\{N_{i}^{h}\right\}$ and $\left\{M_{i}^{h}\right\}$ are the basis functions for the vector quantity $\underline{u}_{\mathrm{a}}$ and the scalar $p$, respectively. A compatible pair of interpolations for primitive variables must be chosen to avoid instabilities usually appearing as oscillations primarily in the pressure field, as dictated by the Ladyzhenskaya-Babuška-Brezzi (LBB) 'inf-sup' stability condition [18,19]. To this end, we employ biquadratic basis functions, $N_{i}(i=1-9)$, to approximate $\underline{u}_{\mathrm{a}}$ and bilinear basis functions, $M_{i}(i=1-4)$, to approximate $p$. Since unequal interpolation (or mixed interpolation) is used, we call the present analysis mixed finite element formulation. Despite the success of avoiding pressure oscillations, this compatibility condition precludes the use of equal-order interpolation for all field variables and, thus, complicates the programming and data handling. As the consideration of mixed approximation method engenders implementation difficulty, Hughes et al. circumvented the need to satisfy the LBB condition by perturbing the pressure test function with a gradient term [20]. This method was later generalized by Hughes and Franca [21] and was proven to have ability to enhance stability of the finite element equation without upsetting consistency. Different variants of equal-order finite element formulations have been reported to circumvent the LBB condition by adding least-square-like terms to the weighted Galerkin method [22-25]. Even the equal-order finite element method has been well-accepted and enjoyed great success in areas of fluid dynamics and heat transfer, we still stick to the mixed finite element formulation mainly because we had no knowledge of circumventing this difficulty in the development of our model starting in the early of 1987.

By adopting standard finite element approach, the assembled matrix equations for a problem having $n^{e}$ elements are derived as $\underline{\underline{A}} \underline{\bar{q}}=\underline{b}$ for the solution vector $\underline{\bar{q}}=\left(u_{j}, v_{j}, p_{j}\right)^{\mathrm{T}}$. The components of the matrix $\underline{\underline{A}}$ are as follows: 


$$
\begin{gathered}
a_{i j}=\sum_{1}^{n^{e}} \int_{\Omega^{h}}\left[\begin{array}{ccc}
C^{i j} & 0 & -M^{j} \frac{\partial N^{i}}{\partial x_{1}}+B^{i} \frac{\partial M^{i}}{\partial x_{1}} \\
0 & C^{i j} & -M^{j} \frac{\partial N^{i}}{\partial x_{2}}+B^{i} \frac{\partial M^{i}}{\partial x_{2}} \\
M^{i} \frac{\partial N^{j}}{\partial x_{1}} & M^{i} \frac{\partial N^{j}}{\partial x_{2}} & 0
\end{array}\right] \mathrm{d} \Omega^{h} \\
+\int_{\Gamma^{h}}\left[\begin{array}{ccc}
-\frac{1}{R e} N^{i} \frac{\partial N^{j}}{\partial x_{k}} \cdot n_{k} & 0 & 0 \\
0 & -\frac{1}{R e} N^{i} \frac{\partial N^{j}}{\partial x_{k}} \cdot n_{k} & 0 \\
0 & 0 & 0
\end{array}\right] \mathrm{d} \Gamma^{h} .
\end{gathered}
$$

In Eq. (20), $C^{i j}$ is expressed as

$$
C^{i j}=\left(N^{i}+B^{i}\right) N^{j} \tilde{V}_{k}^{j} \frac{\partial N^{j}}{\partial x_{k}}+\frac{1}{R e} \frac{\partial N^{i}}{\partial x_{k}} \frac{\partial N^{j}}{\partial x_{k}}-\frac{1}{R e} B^{i} \frac{\partial^{2} N^{j}}{\partial x_{k} \partial x_{k}} .
$$

In the above equation, $\tilde{U}$ and $\tilde{V}$ are evaluated at element centroids. The line integral in (20) involves prescribing essential boundary conditions. Along a boundary where the natural boundary condition is imposed, the vector $\underline{b}$ is given by

$$
\underline{b}=\int_{\Gamma^{h}}\left[\begin{array}{c}
-N^{i} p_{i} n_{1} \\
-N^{i} p_{i} n_{2} \\
0
\end{array}\right] \mathrm{d} \Gamma^{h}
$$

Here, $\left(n_{1}, n_{2}\right)$ denotes the outward unit vector normal to the boundary, at which pressure values are imposed.

In finite element computation of incompressible fluid flows, proper selection of the test space $\underline{w}$ is vital to suppressing node-to-node oscillations primarily in the velocity field. Such oscillations become increasingly significant for high Reynolds number flows and flows with sharp layers in the solution. For this reason, we adopt the Petrov-Galerkin finite element model to enhance the convective stability. This model is regarded as a modification of the Galerkin method in the sense that

$$
B^{i}=\tau N^{j} \tilde{V}_{k}^{j} \partial N^{i} / \partial x_{k}
$$

is added to the basis function $N^{j}$. This engenders favorable consideration of field variables at the upwind side. The parameter $\tau$ shown in Eq. (23) is critical to suppressing oscillatory velocities. In our PetrovGalerkin model, we consider $\tau\left(\equiv \delta \gamma / 2|\underline{u}|^{2}\right)$ as a function of the Peclet number $\gamma(\equiv|\underline{u}| h / 2 \mu)$. Our finite element analysis employs $\delta$, which is analytically derived from the one-dimensional convection-diffusion scalar transport equation in quadratic elements. Depending on the nodal classification, analytical expressions of $\delta$ derived in quadratic elements are as follows [26]:

$$
\delta(\gamma)=\left\{\begin{array}{l}
\frac{1}{2} \operatorname{coth}(\gamma / 2-1 / \gamma) ; \quad \text { center nodes, } \\
\frac{\gamma \sinh \gamma \cosh \gamma-\sinh ^{2} \gamma-4 \cosh \gamma-2 \gamma \sinh \gamma-4}{6 \sinh \gamma \cosh \gamma+\gamma \sinh ^{2} \gamma-6 \sinh \gamma-4 \gamma \cosh \gamma+4 \gamma} ; \quad \text { corner nodes. }
\end{array}\right.
$$

In Eq. (23), we adopt the streamline operator, which was proposed by Brooks and Hughes [27]. This implicitly added artificial damping term in the direction of flow is considered useful not only to stabilize the discrete system, but also to decrease the numerical diffusion error in the multi-dimensional flow analysis. It 
is worth noting that use of this operator has been justified by Johnson and Nävert [28,29] in their convergence analysis of the method. Methods of this class are referred by Johnson and Nävert [28] as streamline diffusion methods and currently by Hughes et al. [30] as Galerkin-least-squares methods.

\section{Finite element calculation of particle trajectory equations}

As in normalization of the flow equations, we can also scale the solid-phase momentum equations. Defining the density ratio

$$
\eta=\frac{\rho_{\mathrm{a}}}{\rho_{\mathrm{p}}}
$$

the dimensionless particle trajectory equation is derived as

$$
\underline{u}_{\mathrm{p}} \cdot \nabla \underline{u}_{\mathrm{p}}=-\eta \nabla p+\frac{3 \eta}{8 r_{\mathrm{p}}}\left|\underline{u}_{\mathrm{a}}-\underline{u}_{\mathrm{p}}\right|\left(\underline{u}_{\mathrm{a}}-\underline{u}_{\mathrm{p}}\right) C_{\mathrm{D}}
$$

This equation can be further rewritten as the convection-reaction equation:

$$
\underline{u}_{\mathrm{p}} \cdot \nabla \underline{u}_{\mathrm{p}}+K \underline{u}_{\mathrm{p}}=\underline{F},
$$

where

$$
\begin{aligned}
& K=\frac{3 \eta C_{\mathrm{D}}}{8 r_{\mathrm{p}}}\left|\underline{u}_{\mathrm{a}}-\underline{u}_{\mathrm{p}}\right|, \\
& \underline{F}=-\eta \nabla p+\underline{u}_{\mathrm{a}} K .
\end{aligned}
$$

The analogy between the above particle trajectory equation and the following transport equation prompts us to develop a convection-reaction finite element model:

$$
a \frac{\partial \phi}{\partial x}+b \frac{\partial \phi}{\partial y}+c \phi=f
$$

To a first approximation, we consider that $a$ and $b$ are two constant values.

In this paper, we are concerned with obtaining good stability and high accuracy for Eq. (30). The goal is that the model presented here provides nodally exact solutions in the one-dimensional case. To this end, we consider first the one-dimensional model equation as follows:

$$
u \frac{\partial \phi}{\partial x}+c \phi=f
$$

We employ in this paper the Petrov-Galerkin finite element model in a domain which is covered with uniform quadratic elements. As is the case when conducting weighted residuals finite element analysis, we should define the basis space $\left\{N_{i}\right\}$ for the field variable $\phi$ and the test space $\left\{W_{i}\right\}$ for Eq. (31). Fig. 1 shows two quadratic elements, each of which has a grid size of $h$. Within the Petrov-Galerkin finite element framework, we add $\frac{1}{2} \alpha h N_{i}^{\prime}$ to $N_{i}$ to obtain the weighting function

$$
W_{i}=N_{i}+\frac{1}{2} \alpha h N_{i}^{\prime} \text {. }
$$

The free parameter $\alpha$ determines the weight placed in favor of the upwind side node. Substituting the quadratic shape functions $\left(N_{1}, N_{2}, N_{3}\right)=((\xi-1) \xi / 2,(1+\xi)(1-\xi),(1+\xi) \xi / 2)$ and the weighting function given in (32) into the weighted residuals statement, we obtain

$$
\sum_{e} \int_{\Omega_{e}} W_{i}\left(u \frac{\partial \phi}{\partial x}+c \phi-f\right) \mathrm{d} x=0 .
$$

In the above, $\xi$, defined as $-1 \leqslant \xi \leqslant 1$, denotes the coordinate for the master element. 

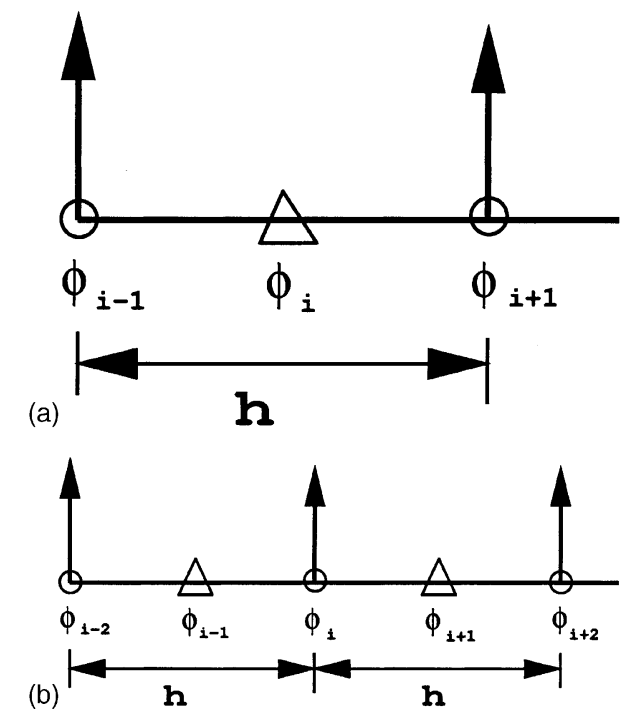

Fig. 1. An illustration of quadratic elements with the uniform grid size $h$. (a) middle node " $\triangle$ " representation; (b) corner node " $\bigcirc$ " representation.

After some algebra, we can obtain discrete equations which are similar to the finite difference equations. Due to space limitations, we will only summarize the results since the derivation is too lengthy to give here. In a quadratic element, we can express the discrete equations at the middle and corner nodes, respectively. The equation for the middle node $i$ shown in Fig. 1(a) is

$$
\left[\frac{1}{5}+\alpha-\frac{1}{\beta}(1+2 \alpha)\right] \phi_{i-1}+4\left(\frac{2}{5}+\frac{\alpha}{\beta}\right) \phi_{i}+\left[\frac{1}{5}-\alpha+\frac{1}{\beta}(1-2 \alpha)\right] \phi_{i+1}=2 \frac{f}{c}
$$

and that for the corner node $i$ shown in Fig. 1(b) is

$$
\begin{gathered}
{\left[\frac{1}{2 \beta}(\alpha+1)-\frac{1}{5}-\frac{\alpha}{2}\right] \phi_{i-2}+2\left[-\frac{1}{\beta}(1+2 \alpha)+\frac{1}{5}+\alpha\right] \phi_{i-1}+\left(\frac{7 \alpha}{\beta}+\frac{8}{5}\right) \phi_{i}} \\
\quad+2\left[\frac{1}{\beta}(1-2 \alpha)+\frac{1}{5}-\alpha\right] \phi_{i+1}+\left[\frac{1}{2 \beta}(-1+\alpha)-\frac{1}{5}+\frac{\alpha}{2}\right] \phi_{i+2}=2 \frac{f}{c} .
\end{gathered}
$$

In the above, the dimensionless parameter $\beta$ is defined as

$$
\beta=\frac{c h}{2 u} \text {. }
$$

Our strategy to determine $\alpha$ is to provide a nodally exact solution $\phi$ in quadratic elements. To achieve this goal, we exploit the following analytic solution for (31) in the derivation:

$$
\phi=C_{1} \mathrm{e}^{-c x / u}+f / c \text {. }
$$

In the above, $C_{1}$ is a constant. By making use of the above analytic $\phi$, we can obtain the exact expressions for $\phi_{i-2}, \phi_{i-1}, \phi_{i}, \phi_{i+1}$ and $\phi_{i+2}$. We then proceed to substitute them into Eqs. (34) and (35) to obtain the analytic $\alpha$ at the middle and corner nodes. After some algebra, the analytic values of $\alpha$ are derived as

$$
\alpha_{\text {middle nodes }}=\frac{4 \beta+\beta \cosh (\beta)-5 \sinh (\beta)}{5(2 \cosh (\beta)-\beta \sinh (\beta)-2)},
$$




$$
\alpha_{\text {corner nodes }}=\frac{8 \beta+4 \beta \cosh (\beta)-2 \beta \cosh (2 \beta)-20 \sinh (\beta)+5 \sinh (2 \beta)}{5(8 \cosh (\beta)-\cosh (2 \beta)-4 \beta \sinh (\beta)+\beta \sinh (2 \beta)-7)} .
$$

We can now extend the analytic one-dimensional analysis to construct a computationally stable and numerically accurate Petrov-Galerkin finite element model for the two-dimensional prototype equation. In this study, we resort to the idea of Brooks and Hughes [27] by adding stabilizing terms along the primary flow direction. The following weighting functions for solving Eq. (30) is, thus, expressed as

$$
W_{i}=N_{i}+\tau\left(a \frac{\partial N_{i}}{\partial x}+b \frac{\partial N_{i}}{\partial y}\right)
$$

or

$$
W_{i}=N_{i}+\tau_{i} \frac{N^{j} \tilde{V}_{k}^{j}}{\sqrt{a^{2}+b^{2}}} \frac{\partial N_{i}}{\partial x_{k}}
$$

where

$$
\tau_{i}=\frac{\sum_{i=1}^{n} \delta\left(\gamma_{Y_{i}}\right) V_{Y_{i}} h_{Y_{i}}}{2 \sqrt{a^{2}+b^{2}}} .
$$

By substituting weighting and shape functions into the weighted residuals statement for Eq. (30), we can derive the algebraic equations on biquadratic elements, shown schematically in Fig. 2. This is followed by deriving the corresponding modified equations at four types of elements. These modified equations are derived and can be expressed in the following general form:

$$
a \frac{\partial \phi}{\partial x}+b \frac{\partial \phi}{\partial y}+c \phi-f=\tau\left\{a c \frac{\partial \phi}{\partial x}+b c \frac{\partial \phi}{\partial y}+a^{2} \frac{\partial^{2} \phi}{\partial x^{2}}+b^{2} \frac{\partial^{2} \phi}{\partial y^{2}}+2 a b \frac{\partial^{2} \phi}{\partial x \partial y}\right\}+S^{\prime} .
$$

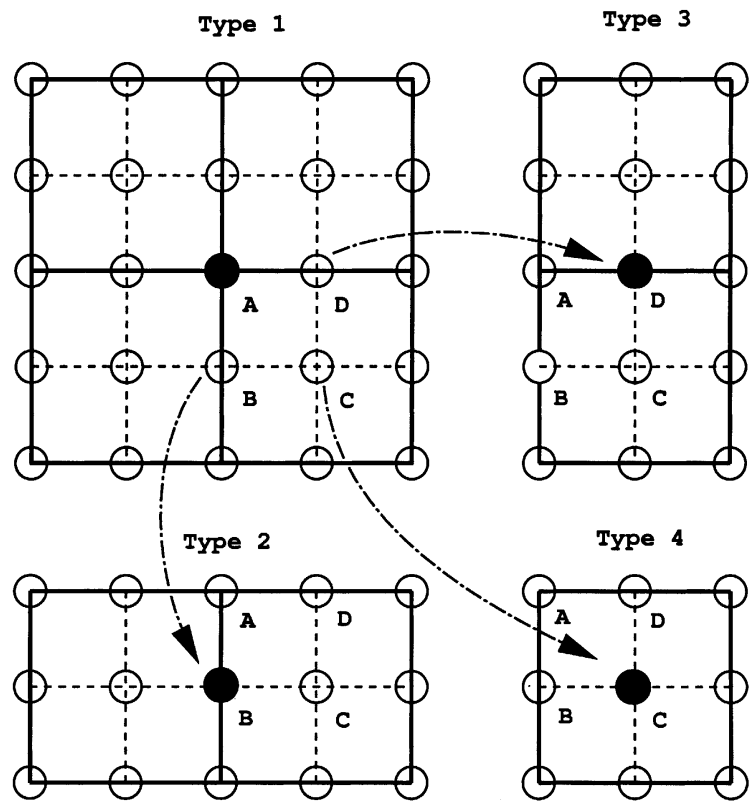

Fig. 2. Four types of element encountered in the biquadratic elements. 
The novelty of the above equation is that the leading dissipative discretization error is added mainly to the flow direction $\theta\left(\equiv \tan ^{-1}(b / a)\right)$. Therefore, the proposed convection-reaction scheme is named as a streamline upwind model owing to the following identity equation:

$$
\frac{\partial^{2} \phi}{\partial s^{2}}=\frac{a^{2}}{a^{2}+b^{2}} \frac{\partial^{2} \phi}{\partial x^{2}}+\frac{2 a b}{a^{2}+b^{2}} \frac{\partial^{2} \phi}{\partial x \partial y}+\frac{b^{2}}{a^{2}+b^{2}} \frac{\partial^{2} \phi}{\partial y^{2}},
$$

where $s$ is the flow direction.

\section{Computed results}

As is typical with other numerical simulations, validation of the two proposed models is the first step towards simulating computationally more difficult problems. The validation of the convection-diffusion equation for the airflow and convection-reaction equation for particle trajectories is accomplished by analyzing the results obtained for the case with analytic data. For more rigorous validation details, refer to [31] for the Navier-Stokes equations. The test on the finite element model developed for convectionreaction equation is given below, followed by considering the clinical problem.

\subsection{Validation tests}

For purposes of validation, we consider first an analytic one-dimensional equation given below:

$$
\frac{\partial \phi}{\partial x}+\phi=1
$$

This equation, subject to the analytic boundary conditions, is amenable to the following exact solution:

$$
\phi=1+3 \mathrm{e}^{-x} .
$$

This problem, defined in $0 \leqslant x \leqslant 1$, is specifically designed to justify the proposed nodally exact convectionreaction finite element model. Solutions are sought in a domain covered with uniform grids. The computed results schematic in Fig. 3 reveal that analytic solutions are reproduced.

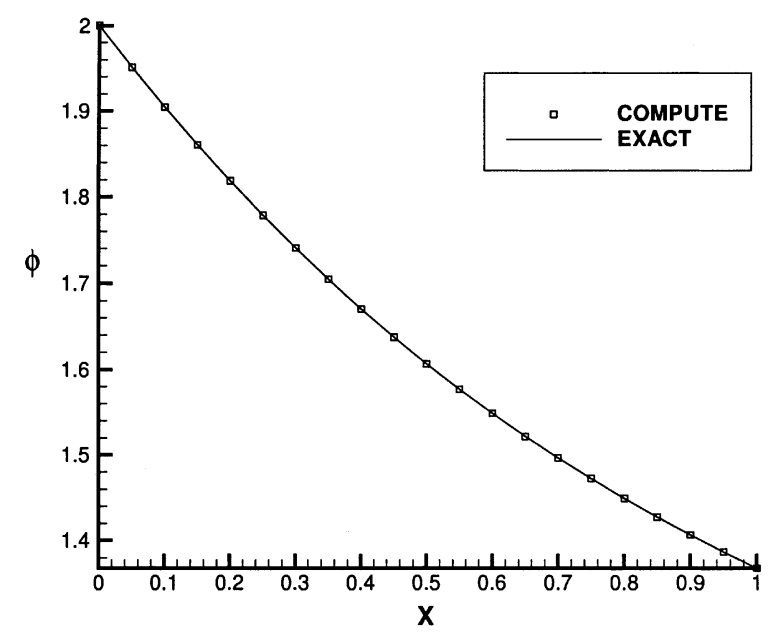

Fig. 3. A comparison of the computed and exact solutions shown in Eq. (46). 
With a view to examining the validity of the streamline upwinding operator used in multi-dimensional analyses, we have undertaken a numerical analysis of the following scalar convection-reaction equation in a domain of $0 \leqslant x, y \leqslant 1$ :

$$
u \frac{\partial \phi}{\partial x}+v \frac{\partial \phi}{\partial y}+c \phi=f
$$

The coefficients $u, v$ and $c$ are chosen $u=2 x+y+1, v=x+2 y+1$ and $c=x+y+1$. Here, the source term $f$ is chosen as $f=x^{3}+2 x^{2} y+2 x y^{2}+y^{3}+6 x^{2}+9 x y+6 y^{2}+4 x+4 y+1$ to make the test problem (47) amenable to the exact solution given below:

$$
\phi=x^{2}+x y+y^{2}+1 \text {. }
$$

Finite element solutions are sought in a square covered with uniform grids. We start from a very coarse grid, say $5 \times 5$, and proceed to continuously refined grids $10 \times 10,20 \times 20$ and $40 \times 40$. For this study, numerical errors are computed and cast in their $L_{2}$ norms. As Table 1 reveals, the computed errors are continuously reduced with an increase of the grid number. It is thus instructive to plot $c=\log \left(\operatorname{err}_{1} /\right.$ $\left.\operatorname{err}_{2}\right) / \log \left(M_{2} / M_{1}\right)$, from which it is known that the rate of convergence for the proposed scheme is 3.9 (Fig. 4). Here, $\operatorname{err}_{i}(i=1,2)$ denotes $L_{2}$-error norms computed at two continuously refined grids, $\left(M_{1}+1\right)^{2}$ and $\left(M_{2}+1\right)^{2}$. Through above two analytic tests, we have confidence to simulate the solid-phase equation.

Table 1

The computed $L_{2}$, error norms and the rates of convergence

\begin{tabular}{lll}
\hline No. of elements & $L_{2}$ norm & Convergent rate \\
\hline $5 \times 5$ & $6.119 \times 10^{-6}$ & 3.87 \\
$10 \times 10$ & $4.196 \times 10^{-7}$ & 3.93 \\
$20 \times 20$ & $2.748 \times 10^{-8}$ & 3.97 \\
$40 \times 40$ & $1.758 \times 10^{-9}$ & \\
\hline
\end{tabular}

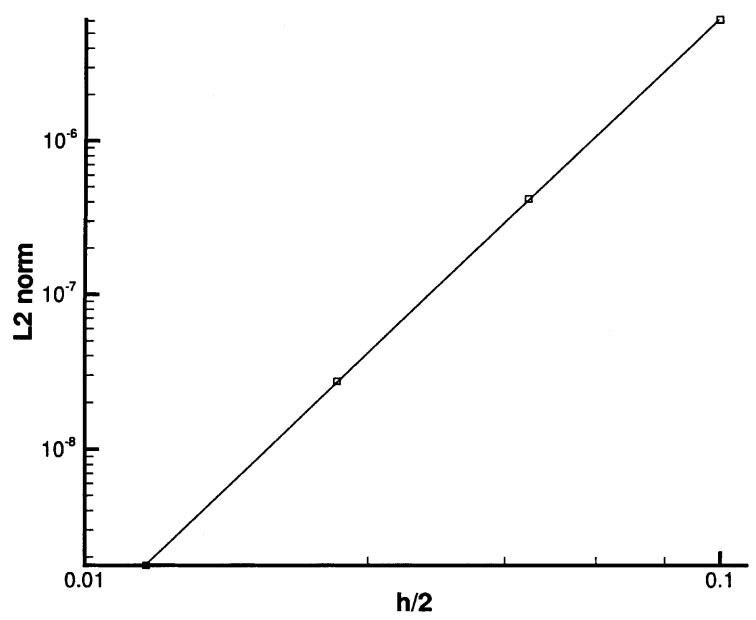

Fig. 4. The plot of $L_{2}$ norms against the grid spacings for showing the rate of convergence. 


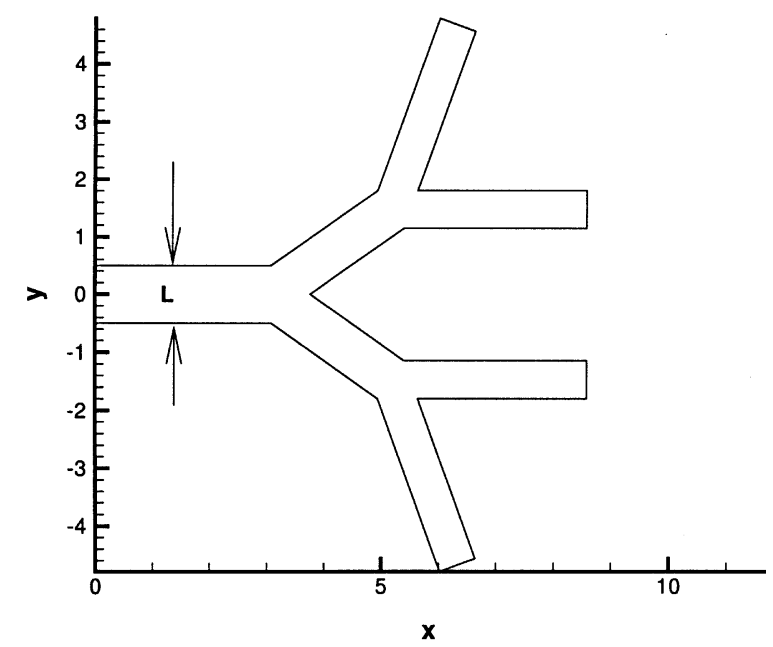

Fig. 5. The outline of the airways under the present investigation.

\subsection{Weibel model of the human central airway}

The problem, known as the Weibel model of the human central airway, has been considered by Wilquem and Degrez [1]. The domain, schematically shown in Fig. 5, involves a mother branch and a set of symmetrically configured lateral and medial branches. Downstream of the mother branch, there exist two bifurcation points which are symmetric to the mother branch centerline. The principal dimensions and the turning angles for this test model are also shown in the schematic diagram. In this study, the full domain was computed in order to avoid the Coanda effect present even in a symmetric configuration.

Computations for this problem were carried out for air with a kinematic viscosity of $v(\equiv 1.505 \times$ $\left.10^{-5} \mathrm{~m}^{2} / \mathrm{s}\right)$. The diameter of the mother branch $L\left(\equiv 3.5 \times 10^{-3} \mathrm{~m}\right)$ was chosen as the characteristic length. In this investigation, the characteristic velocity $U(\equiv 0.86 \mathrm{~m} / \mathrm{s})$ was chosen as the mean inlet velocity, which is parabolic in form. The resulting Reynolds number $\operatorname{Re}(\equiv U L / v)$ was 200 . The dimension investigated in this model corresponds to those encountered in the fifth to seventh generations of the human central airways. The pressure values at the exit plane of the medial and lateral branches were all set to be one.

In the finite element method, we are permitted to construct grids on a block-by-block basis. We divide the whole domain into 14 blocks, as shown in Fig. 6(a). This facilitates grid generation in each block, thus helping to retain grid smoothness. Grids are also clustered near the no-slip wall as well as in the vicinity of bifurcation. The resulting grid topology is shown in Fig. 6(b), which involves using 11991 nodal points and 2900 biquadratic elements. Finite element analysis of flow in the Weibel model starts with simulating Navier-Stokes equations subject to the boundary conditions described above. The computed flowfield, characterized by $\left(u_{\mathrm{a}}, v_{\mathrm{a}}\right)$ and $p$, is considered as the source term for solving the particle velocity governed by Eq. (6). Since they are influential factors in determining particle trajectories, we plot streamlines and pressure contours in Figs. 7 and 8, respectively, for the airflow. The characteristic features of the branching flow, such as curved streamlines, stagnation points and flow reversal, are clearly seen in Fig. 7.

Upon obtaining the values of $\underline{u}_{\mathrm{a}}$ and $p$ for the airflow, we can solve for $u_{\mathrm{p}}$ and $v_{\mathrm{p}}$ using Eq. (6) and the proposed streamline upwind convection-reaction finite element model. The resulting motions for particles with radii of $r_{\mathrm{p}}=2.5 \times 10^{-3} \mathrm{~L}$ and $2.5 \times 10^{-4} \mathrm{~L}$ are best depicted by their particle tracers schematically shown in Fig. 9. Discussion of inhaled particle motions must consider the interphase drag $C_{p i}\left(\underline{u}_{\mathrm{a}}-\underline{u}_{\mathrm{p}}\right)$ and 

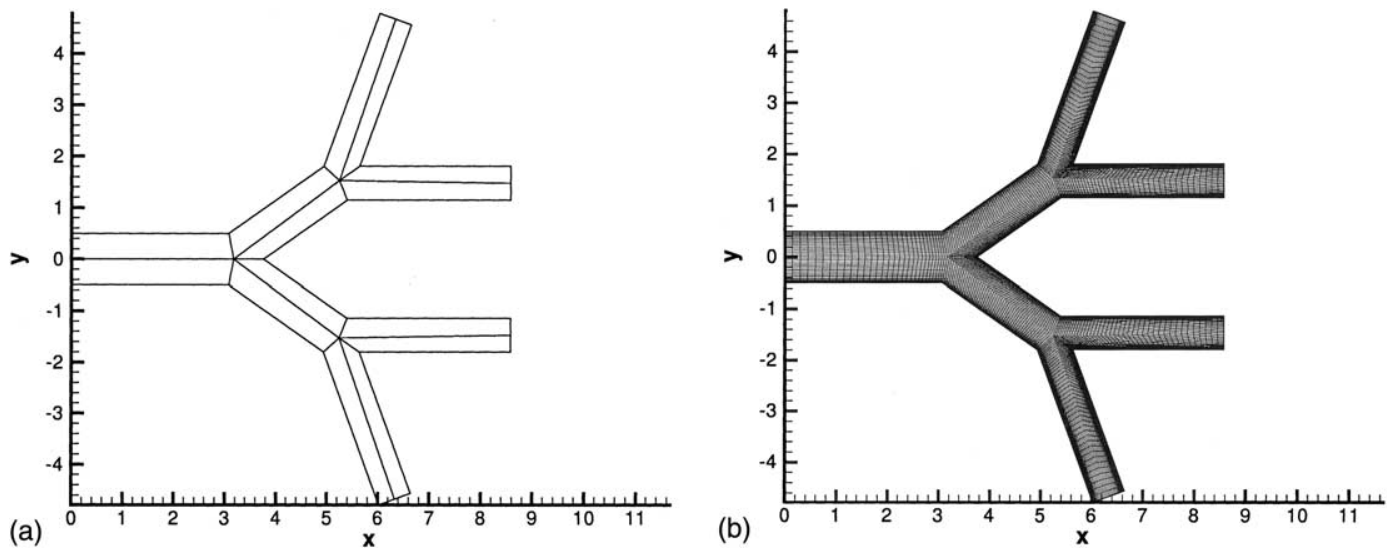

Fig. 6. Blocks and grid distribution.

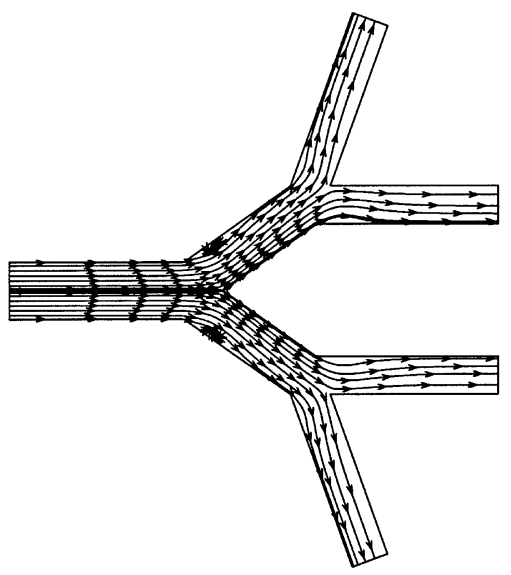

Fig. 7. Streamline plots for the airflow.

the pressure gradient $-\left(1 / \rho_{\mathrm{p}}\right) \nabla p$ shown on the left-hand side of Eq. (6). A check of the magnitudes of these driving forces reveals that the interface drag considerably dominates the pressure gradient established in the airflow. This implies that the interface drag between the particle and gas phases plays the dominant role in the equations of motion for the inhaled particles. To better understand the particle motion, we need to focus our attention on the computed values of $C_{p i}\left(u_{\mathrm{a}}-u_{\mathrm{p}}\right)$ and $C_{p i}\left(v_{\mathrm{a}}-v_{\mathrm{p}}\right)$. According to Eq. (10) for $C_{p i}$, Eq. (8) for $C_{\mathrm{D}}$, and Eq. (9) for $R e_{\mathrm{p}}, C_{p i}$ can be expressed as $C_{p i}=C_{1} r_{\mathrm{p}}^{-2}+C_{2} r_{\mathrm{p}}^{0.313}$, where $C_{1}=\frac{9}{2}(\eta / R e)$ and $C_{2}=0.15 C_{1}\left(2 R e\left|\underline{u}_{\mathrm{a}}-\underline{u}_{\mathrm{p}}\right|^{0.687}\right)$. Since $\eta$ and $R e$ are both positive values, $C_{p i}>0$ throughout the flow. Whether the inhaled particle is subjected to an accelerating or decelerating force depends, therefore, on the sign of $\underline{u}_{\mathrm{a}}-\underline{u}_{\mathrm{p}}$. This motivates us to plot in Fig. 10 contour values of $u_{\mathrm{a}}-u_{\mathrm{p}}$ and $v_{\mathrm{a}}-v_{\mathrm{p}}$. For particles entering the trachea vessel, they are carried by airflow, with a negligibly small velocity difference. Downstream of the mother branch, the airflow is decreased by the established large adverse pressure gradient, as seen in Fig. 8, at the first bifurcation point. As Fig. 10(a) shows, $u_{\mathrm{a}}<u_{\mathrm{p}}$ in the vicinity of the stagnation point; thus, a negative $x$-component interface drag is applied to approaching solid particles. It is also found that $v_{\mathrm{a}}>v_{\mathrm{p}}$ on the upper branch, and that $v_{\mathrm{a}}<v_{\mathrm{p}}$ on the lower branch. This indicates that a positive 


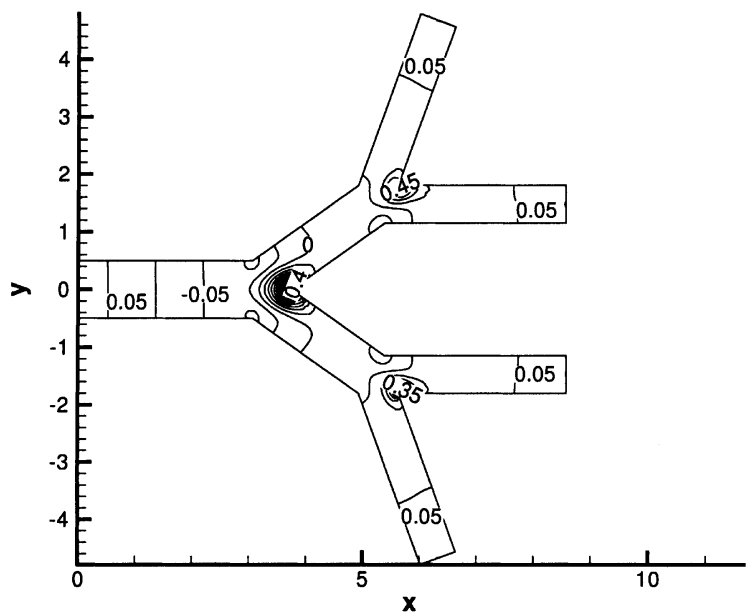

Fig. 8. Pressure contour plots for the airflow.
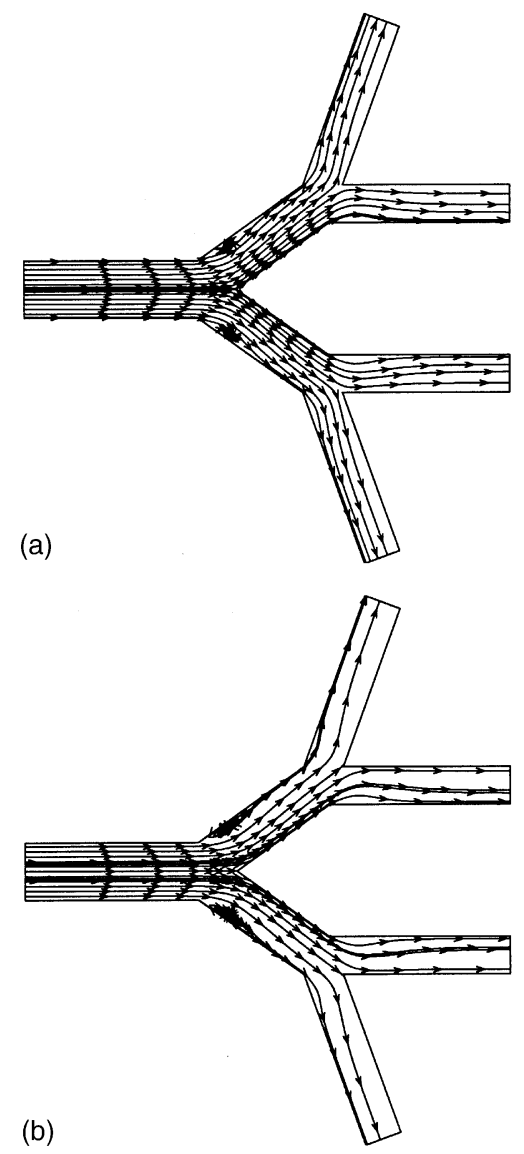

Fig. 9. Particle tracer plots for the inspiratory particles (a) $r_{\mathrm{p}}=2.5 \times 10^{-3} L$; (b) $r_{\mathrm{p}}=2.5 \times 10^{-4} L$. 
$y$-component interface drag is applied to the $y$-momentum equation for particles turning towards the upper branch of the vessel. On the other hand, a negative $y$-component interface drag is applied to particles turning towards the lower branch of the vessel. This explains why inhaled particles bend in the way shown in Fig. 9. Particles proceeding further downstream change direction due to the interface drag which arises due to the difference between $u_{\mathrm{a}}-u_{\mathrm{p}}$ and $v_{\mathrm{a}}-v_{\mathrm{p}}$. As a means of determining the particle motion, we plot contour values of $C_{p i}\left(u_{\mathrm{a}}-u_{\mathrm{p}}\right)$ and $C_{p i}\left(v_{\mathrm{a}}-v_{\mathrm{p}}\right)$ in Fig. 11. A large change in interface drag is seen near the bifurcation points.

For particles of different radii $r_{\mathrm{p}}$ in the same flow field, it is found that the smaller $r_{\mathrm{p}}$ is, the larger is $C_{p i}$. This indicates that the interface drag has a prevailing influence on the particle motion. Under these circumstances, particles have a tendency to change their trajectories to adapt to the airflow. To confirm this, we plot in Fig. 12 velocity profiles at four selected sections for particles with $r_{\mathrm{p}}=2.5 \times 10^{-3} L$ and
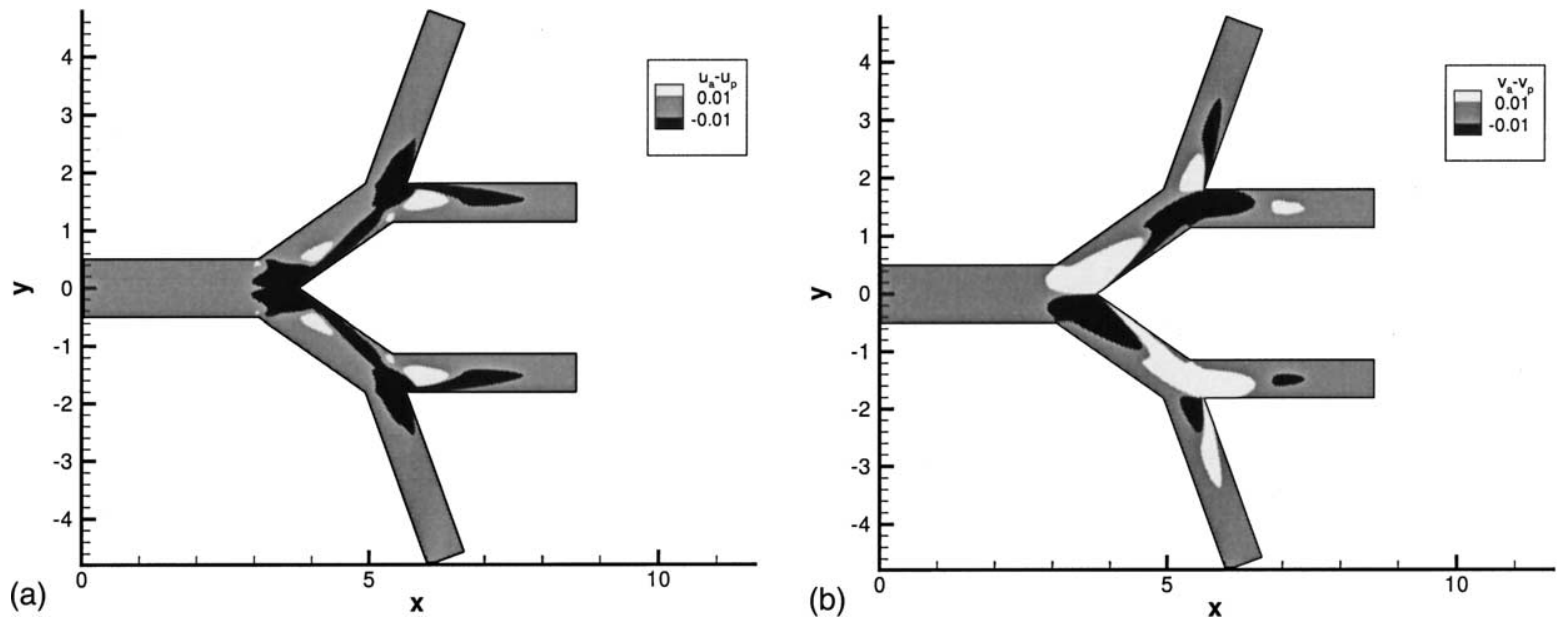

Fig. 10. The contour plot of the velocity difference between air and particles, for the case of $r_{\mathrm{p}}=2.5 \times 10^{-3} L$ (a) in the $x$-direction $\left(u_{\mathrm{a}}-u_{\mathrm{p}}\right)$; (b) in the $y$-direction $\left(v_{\mathrm{a}}-v_{\mathrm{p}}\right)$.
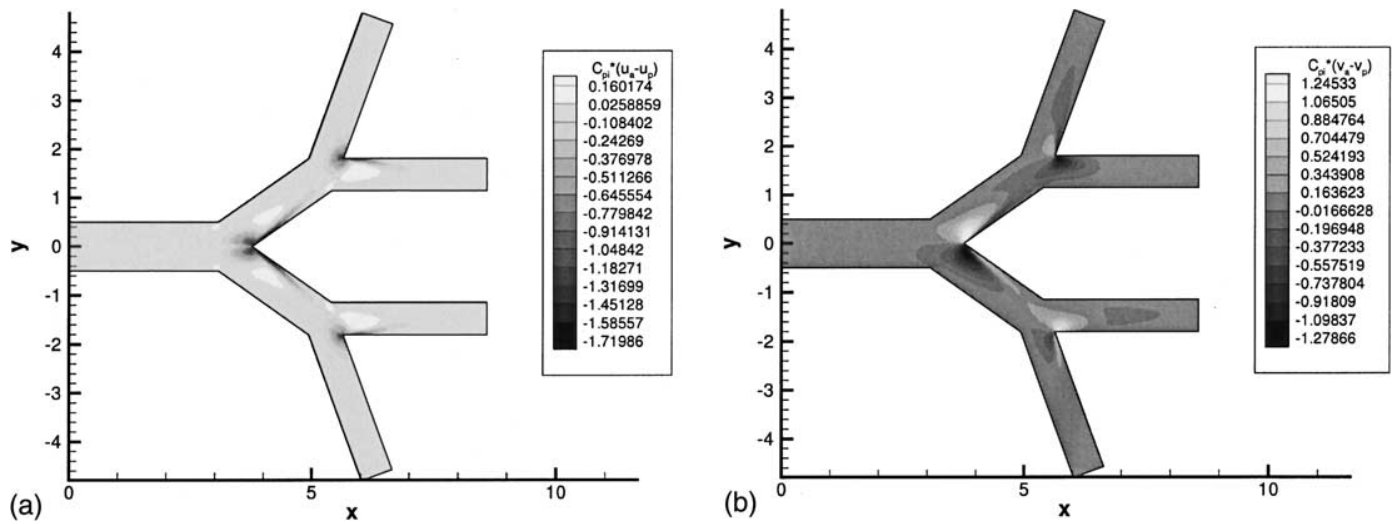

Fig. 11. The contour plot of $C_{p i}\left(\underline{u_{\mathrm{a}}}-\underline{u_{\mathrm{p}}}\right)$ for the case of $r_{\mathrm{p}}=2.5 \times 10^{-3} L$. (a) $C_{p i}\left(u_{\mathrm{a}}-u_{\mathrm{p}}\right)$; (b) $C_{p i}\left(v_{\mathrm{a}}-v_{\mathrm{p}}\right)$. 

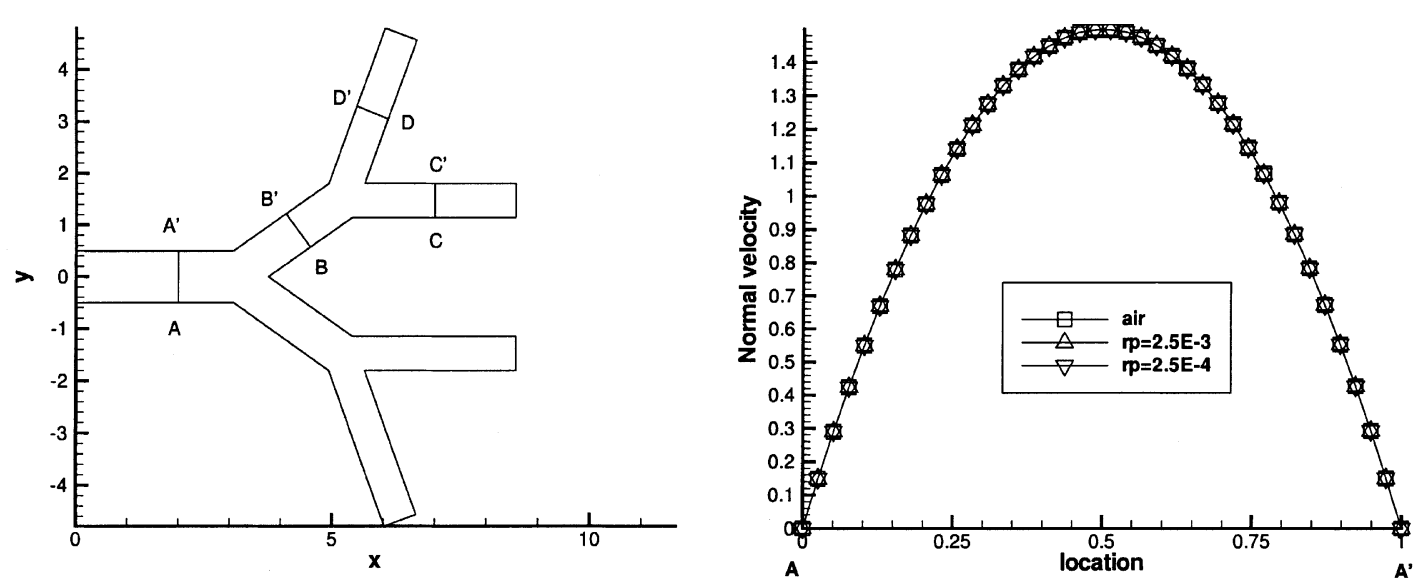

(a)

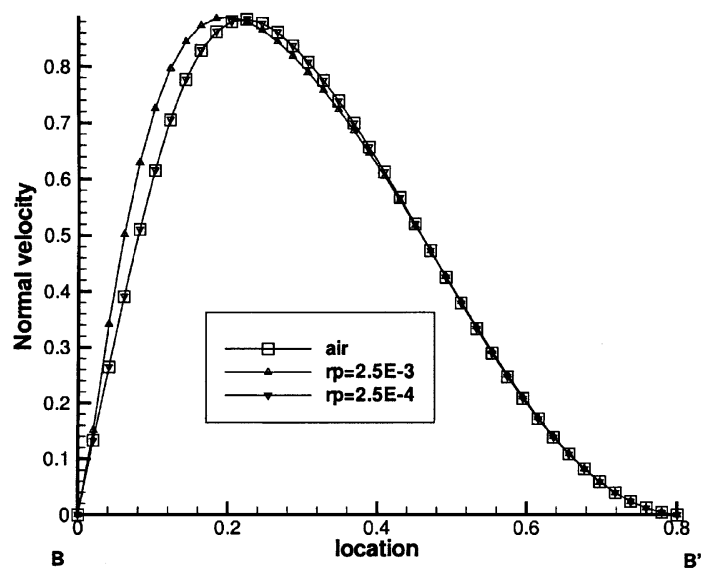

(b)

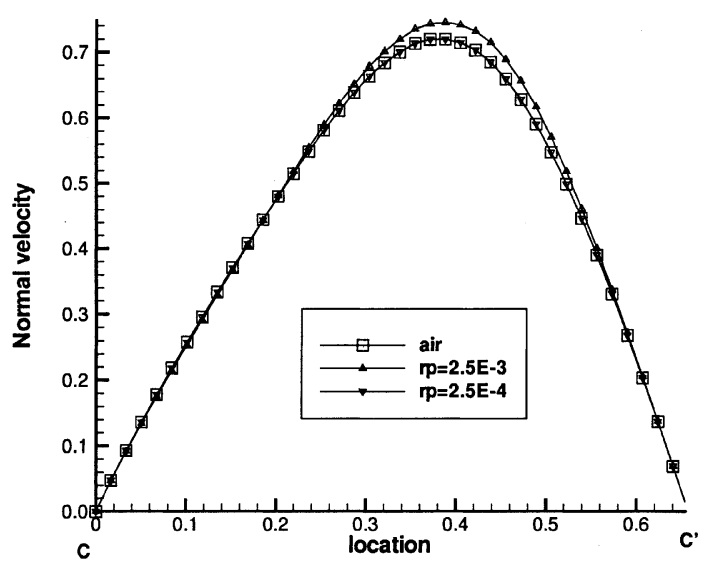

(c)

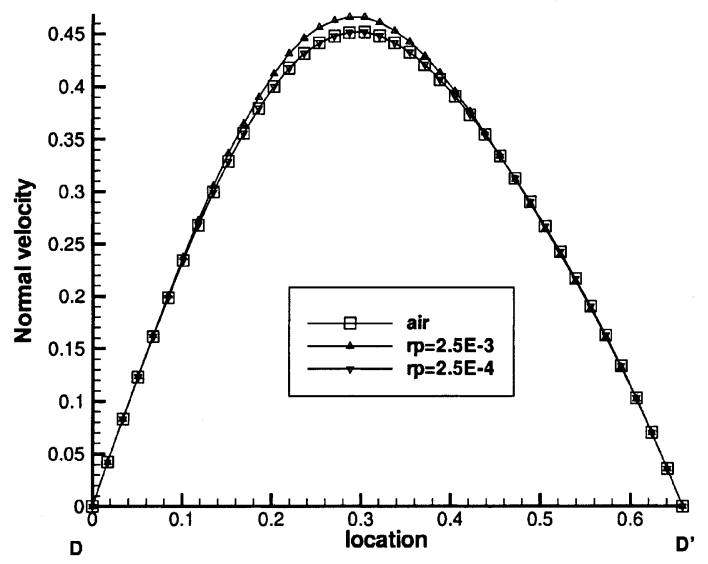

(d)

Fig. 12. The normal velocity profiles plotted at several cross sections $\mathrm{AA}^{\prime} \sim \mathrm{DD}^{\prime}$ for the case of $r_{\mathrm{p}}=2.5 \times 10^{-3} L$. 
$2.5 \times 10^{-4} \mathrm{~L}$. It is seen that the particle velocity profile approaches that of the airflow for the case with a smaller particle radius.

\section{Concluding remarks}

We have presented in this paper a two-phase flow model for predicting inhaled particles in the human airway. Under the rational assumption that the porosity of the solid phase is negligibly small when compared with its gas-phase counterpart, the equations governing the two-phase flow are coupled through gasphase interface drag, which, as usual, is associated with the relative velocity of the two phases. The test problem of clinical relevance involved geometric bifurcation points in the air vessel. The feature worthy of note is that the upwinding added to improve stability is determined exactly on the nodal basis in both onedimensional prototype equations for the gas and solid phases. The representative equation for the gas phase is the convection-diffusion equation. As for the solid phase, the model equation for the transport of inhaled particles is the convection-reaction equation. A guideline for extending one-dimensional nodally exact finite element models for solid and gas phases is to use a streamline operator in the multi-dimensional analysis of two-phase equations. The implicitly added stabilizing term enhances the finite element equations while the prediction accuracy is retained without deterioration due to numerical crosswind diffusion errors. Having developed the streamline upwind finite element models for solving the coupled system of continuitymomentum equations for the gaseous equations and the equations of motion for the solid phase, we applied them iteratively to solve for two-phase equations through the solid-gas interface drag. As is usual, this interface drag is a function of the relative velocity. The resulting two-phase finite element models were applied in combination to obtain the solution for the human central airway, with an aim at revealing the particle trajectory and the deposition sites of inhaled particles.

\section{References}

[1] F. Wilquem, G. Degrez, Numerical modeling of steady inspiratory airflow through a three-generation model of the human central airways, J. Biomech. Engrg. 119 (1997) 59-65.

[2] S.F. Tsai, T.W.H. Sheu, T.M. Chang, Lung effect on the hemodynamics in pulmonary artery, Int. J. Numer. Meth. Fluids 36 (2001) 249-263.

[3] T.W.H. Sheu, S.F. Tsai, W.S. Hwang, T.M. Chang, A finite element study of the blood flow in total cavopulmonary connection, Comput. Fluids 28 (1) (1999) 19-39.

[4] D.L. Swift, Use of mathematical aerosol deposition models in predicting the distribution of inhaled therapeutic aerosols, in: A.J. Hickey (Ed.), Inhalation Aerosols: Physical and Biological Basis for Theory, Marcel Decker, Inc., New York, 1996, pp. 17-34.

[5] I.M. Katz, B.M. Davis, T.B. Martonen, A numerical study of particle motion within the human larynx and trachea, J. Aerosol Sci. 30 (2) (1999) 173-183.

[6] T.B. Martonen, I.M. Katz, Deposition patterns of aerosolized drugs within human lungs; effects of ventilatory parameters, Pharm. Res. 10 (1993) 871-878.

[7] P. Corieri, Experimental and numerical investigation of flows in bifurcations within lung airways, Ph.D. Thesis, University Libre de Bruxelles, 1994.

[8] A. Favre, Equations des gaz turbulents compressibles, J. Mechanique 4 (1965) 361.

[9] T.W.H. Sheu, S.M. Lee, M.Y. Chen, V. Yang, Analysis of combustion processes in a mobile granular propellant bed, AIAA Progr. Astronautics Aeronautics 154 (1993) 477-490.

[10] T.W.H. Sheu, S.M. Lee, Numerical study of two-dimensional solid-gas combustion through granulated propellants, Int. J. Numer. Heat Transf. Part A: Appl. 27 (4) (1995) 395-415.

[11] T.W.H. Sheu, S.M. Lee, Analysis of combustion processes in a gas interior ballistics, Int. J. Comput. Fluid Dynamics 4 (1995) $57-71$.

[12] G.B. Wallis, One Dimensional and Two Phase Flows, McGraw-Hill, New York, 1969.

[13] K.K. Kuo, J.H. Koo, T.R. Davis, G.R. Coates, Transient combustion in mobile gas-permeable propellants, Acta Astronautica 3 (1976) 573-591. 
[14] A.S. Goharrizi, M. Taheri, J. Fathikalajahi, Prediction of particle deposition from a turbulent stream around a surface-mounted ribbon, Aerosol Sci. Technol. 29 (1998) 141-151.

[15] R. Cliff, W.H. Gauvin, Motion of entrained particles in gas streams, Canad. J. Chem. Engrg. 49 (1971) 439.

[16] P.G. Saffman, The lift on a small sphere in a slow shear flow, J. Fluid Mech. 22 (1965) 385-398.

[17] G. Ahmadi, D.H. Smith, Particle transport and deposition in a hot-gas cleanup pilot plant, Aerosol Sci. Technol. 29 (1998) 183-205.

[18] F. Brezzi, On the existence, uniqueness and approximation of saddle point problems arising from Lagrangian multipliers, RAIRO, Anal. Numer. 8 (R2) (1974) 129-151.

[19] I. Babuška, Error bounds for finite element methods, Numer. Math. 16 (1971) 322-333.

[20] T.J.R. Hughes, L.P. Franca, M. Balestra, A new finite element formulation for computational fluid dynamics: V. Circumventing the Babuška-Brezzi condition: A stable Petrov-Galerkin formulation of the Stokes problem accommodating equal-order interpolations, Comput. Meth. Appl. Mech. Engrg. 59 (1986) 85-99.

[21] T.J.R. Hughes, L.P. Franca, A new finite element formulation for computational fluid dynamics: VII. The Stokes problem with various well-posed boundary conditions: Symmetric formulations that converge for all velocity/pressure spaces, Comput. Meth. Appl. Mech. Engrg. 65 (1987) 85-96.

[22] B.N. Jiang, V. Sonnad, Least-squares solution of incompressible Navier-Stokes equations with the $p$-version of finite elements, NASA Tech. Mem. 105203, ICOMP-91-14, September, 1991.

[23] D. Winterscheidt, K.S. Surana, $p$-Version least squares finite element formulation for two-dimensional incompressible fluid flow, Int. J. Numer. Meth. Fluids 18 (1994) 43-69.

[24] J. Douglas Jr., J. Wang, An absolutely stabilized finite element method for the Stokes problem, Math. Comput. 52 (186) (1989) 495-508.

[25] T.E. Tezduyar, S. Mittal, S.E. Ray, R. Shih, Incompressible flow computations with stabilized bilinear and linear equal-orderinterpolation velocity-pressure elements, Comput. Meth. Appl. Mech. Engrg. 95 (1992) 221-242.

[26] T.W.H. Sheu, H.Y. Shiah, A new finite element model for simulating viscoelastic fluid flow, Int. J. Numer. Meth. Heat Fluid Flow, submitted for publication.

[27] A.N. Brooks, T.J.R. Hughes, Streamline upwind Petrov-Galerkin formulations for convection-dominated flows with particular emphasis on the incompressible Navier-Stokes equations, Comput. Meth. Appl. Mech. Engrg. 32 (1982) 199-259.

[28] C. Johnson, U. Nävert, An analysis of some finite element methods for advection-diffusion problems, in: O. Axelsson, L.S. Frank, A. Van Der Sluis (Eds.), Analytical and Numerical Approaches to Asymptotic Problems in Analysis, North-Holland, Amsterdam, 1981, pp. 99-116.

[29] U. Nävert, A finite element method for convection-diffusion problem, Ph.D. Thesis, Department of Computer Science, Chalmers University of Technology, Göteborg, Sweden 1982.

[30] T.J.R. Hughes, L.P. Franca, G.M. Hulbert, A new finite element formulation for computational fluid dynamics: VIII. The Galerkin/least-squares method for advective-diffusive equations, Comput. Meth. Appl. Mech. Engrg. 74 (1989) $41-54$.

[31] T.W.H. Sheu, S.F. Tsai, M.M.T. Wang, A Petrov-Galerkin formulation for incompressible flows at high Reynolds numbers, Int. J. Comput. Fluid Dynamics 5 (1995) 213-230. 